\title{
Outcome of Pregnancy Complicated by Obstetric Cholestasis
}

\author{
Nishaํ, Roopam Singh², Kumari Archana ${ }^{3}$ \\ ${ }^{1}$ Department of Obstetrics and Gynaecology, Bokaro General Hospital, Bokaro, Jharkhand, India. ${ }^{2}$ Department of \\ Obstetrics and Gynaecology, Bokaro General Hospital, Bokaro, Jharkhand, India. ${ }^{3}$ Department of Obstetrics and \\ Gynaecology, Bokaro General Hospital, Bokaro, Jharkhand, India.
}

\section{ABSTRACT}

\section{BACKGROUND}

Pruritus is the primary clinical symptom of ICP. It may considerably impair the patient's quality of life causing sleep deprivation, psychological suffering and even suicidal thoughts. Pruritus is most severe in the evening, with a predilection for the palms of the hands and soles of the feet, and is not associated with any specific skin lesions. The main biochemical alterations are elevations of serum bile acids and aminotransferase activities. We wanted to study the outcome of pregnancy complicated by obstetric cholestasis in terms of maternal and foetal outcome.

\section{METHODS}

This is a prospective study done over a period of 18 months in 80 booked antenatal patients who complained of pruritus during their pregnancy. They were followed, and maternal and perinatal outcomes recorded. Appropriate statistical analysis was used for result.

\section{RESULTS}

Incidence of obstetric cholestasis in our hospital was 5\%. Majority of the patients were primigravida (60\%) and presented at gestational age between 32-36 weeks. Maternal morbidities are due to sleep disturbance (68.75\%), dyslipidaemia (48.75\%), deranged coagulation profile (22.5\%), PPH (8.75\%), increased operative interference $(57.5 \%)$ and PROM (23.75\%). Perinatal outcomes were MSL (23.75\%), prematurity $(25 \%)$, stillbirth $(2.5 \%)$, NICU admission $(18.75 \%)$, foetal distress $(20 \%)$ and LBW (18.75\%). Maximum number of patients were delivered between 37-38 weeks.

\section{CONCLUSIONS}

Active and timely intervention is needed to avoid unnecessary and early intervention and prevent the risk of prematurity.

\section{KEY WORDS}

Obstetric Cholestasis, Intrahepatic Cholestasis of Pregnancy, Foetal Distress, Ursodeoxycholic Acid, Pruritus in Pregnancy, Sleep Disturbance in Pregnancy
Corresponding Author: Dr. Nisha,

D/o. Rameshwar Prasad,

Shiv Govind Sah Lane,

Dahiyawan, Chapra-13

E-mail: preety.nisha22@gmail.com

DOI: $10.14260 / j e m d s / 2019 / 776$

Financial or Other Competing Interests: None.

How to Cite This Article:

Nisha, Singh R, Archana K. Outcome of pregnancy complicated by obstetric cholestasis. J. Evolution Med. Dent. Sci. 2019;8(48):3593-3598, 10.14260/jemds/2019/776

Submission 10-06-2019, Peer Review 10-11-2019, Acceptance 19-11-2019, Published 02-12-2019. 


\section{BACKGROUND}

Intrahepatic cholestasis of pregnancy (ICP) or obstetric cholestasis (OC), is the most common liver disorder during pregnancy. It normally presents in the second half of pregnancy, ${ }^{1}$ typically to recur in subsequent pregnancies and is associated with significantly high adverse maternal and foetal outcome. ${ }^{2}$ The aetiology is complex and appears to be heterogenous $^{3}$ possibly with hormonal, genetic, environmental and dietary influences. It usually presents in the third trimester, after 30 weeks of gestation, but rare cases developing as early as 6 to 10 weeks ${ }^{4}$ have been described.The prevalence varies according to geographic location $^{2}$ and ethnicity. The incidence of obstetric cholestasis among Indian women has been reported to be about 1\%.5,6

Pruritus is the primary clinical symptom of ICP. It may considerably impair the patient's quality of life causing sleep deprivation, psychological suffering and even suicidal thoughts. Pruritus is most severe in the evening, with a predilection for the palms of the hands and soles of the feet, and is not associated with any specific skin lesions. The main biochemical alterations are elevations of serum bile acids and aminotransferase activities. Serum total bile acid level may increase 10-100 times above the normal range and higher foetal complications rates were observed with maternal fasting bile acid level exceeding $40 \mu \mathrm{mol} / \mathrm{L}^{7}$ Obstetric cholestasis is a stressful condition for mothers and is associated with significant maternal morbidities.They have an increased risk for postpartum haemorrhage. There is also increased risk for preterm labour and operative interference.

Intrahepatic Cholestasis of pregnancy can have devastating consequences for the foetus. Adverse foetal outcomes associated with the condition include preterm labour, preterm prelabour rupture of membrane, foetal distress, abnormal CTG, meconium staining, spontaneous intrauterine death. 4,8 Ursodeoxycholic Acid is the drug mainly used for relief of pruritus and improvement of the liver dysfunction, and is very effective in treating this condition.

There is considerable debate on extent of maternal and foetal risks in different studies done on outcome of pregnancies complicated by $\mathrm{OC}$ and in our hospital no studies has been done to study the demographic variation and effect of obstetric cholestasis on maternal and foetal outcome hence this study is chosen.

\section{METHODS}

The study was prospective study conducted in department of Obstetrics and Gynaecology, Bokaro General Hospital, a tertiary care centre in Bokaro District of Jharkhand over a period of One and a half year( June 2016 to Dec 2017). 80 Pregnant women with complain of pruritus in late second \& third trimester with specific predilection to palms and soles and in night were included with exclusion to other liver disorder.

Sample size was calculated for continuous outcome and means by the statistical formula as follow;

$$
\begin{aligned}
& \mathrm{n}=\left\{\mathrm{z}^{2} \mathrm{p}(1-\mathrm{p})\right\} / \mathrm{d}^{2} \\
& \mathrm{~N} \text { (Total population) }=3609 \text { per year (Approx.) } \\
& \mathrm{n}=\text { sample size for current study }
\end{aligned}
$$

$\mathrm{z}=\mathrm{Z}$ is standard normal variate at level of significance (i.e. 1.96 for $95 \%$ confidence level)

$\mathrm{p}=$ Expected prevalence or proportion $=5.5 \%=0.055$

(taken from previous year hospital data)

$\mathrm{d}=$ Precision $=0.05$ (Type 1 error)

Therefore,

$\mathrm{n}=\left\{\mathrm{z}^{2} \mathrm{p}(1-\mathrm{p})\right\} / \mathrm{d}^{2}=\left\{(1.96)^{2}(0.055)(1-0.055)\right\} /(0.05)^{2}$ $=79.87 \approx 80$

Hence the required sample size is 80 .

Patients were informed about the purpose of study and aim of study and after taking informed consent included in study. Through history was elicited and clinical examination is done. Lab investigation was done like Liver Function Test, Prothrombin Time International Normalized Ratio and Lipid Profile to see pre-treatment value. Due to some constrain, Serum bile acid testing was not done in present study and serum transaminases level were used to follow the patients.

LFT was repeated weekly or fortnightly depending on transaminases value. All patients included in study has been given Ursodeoxycholic acid 300-1200 mg/day in divided doses for the rest of antenatal period. Labour and delivery events of these women is monitored and recorded properly along with postpartum and perinatal periods. Women are followed at 6 weeks of post-partum period for resolution of symptoms as well as for normal or altered LFT. The Frequency of obstetric cholestasis in the hospital, the Period of Gestation at which it appears, and the Relationship of Obstetric Cholestasis with Maternal Age and Parity were studied.

Maternal Outcomes were studied in reference to Sleep Disturbance due to severe pruritus, dyslipidaemia ${ }^{9}$, Deranged Coagulation Profile (increased PT), PROM, Mode Of Delivery vaginal delivery, forceps application, elective and emergency caesarean section, Postpartum Haemorrhage. Foetal Outcome were studied in reference to Foetal Distress (defined as either foetal bradycardia $<100 \mathrm{bpm}$ or foetal tachycardia $>160$ bpm), Meconium Stained Liquor, Preterm Birth (delivery before 37 weeks of gestation), Low Birth Weight (birth weight $<2.5 \mathrm{Kg}$ ), NICU admission rate and Perinatal Death (IUFD/Still born). Postpartum resolution was studied in reference to improvement of pruritus and abnormal Liver Function Tests after 6 weeks of delivery.

\section{Statistical Analysis}

Statistical analysis was carried out in present study by using SPSS version and Microsoft Excel 2010 for graphical representation. The data are presented as Mean \pm SD, $95 \%$ confidence interval or as percentage where appropriate. A "pvalue" should be considered to be no significant if $>0.05$ and significant if $<0.05$. Mean, standard deviation and variance

\begin{tabular}{|c|c|c|}
\hline Parameter & No. of Patients (n) & Percentage \\
\hline with obstetric cholestasis & 183 & $5.07 \approx 5 \%$ \\
\hline without obstetric cholestasis & 3426 & $94.93 \approx 95 \%$ \\
\hline Total deliveries & 3609 & $100 \%$ \\
\hline
\end{tabular}
was calculated by Chi square test and proportion test.

\section{RESULTS}




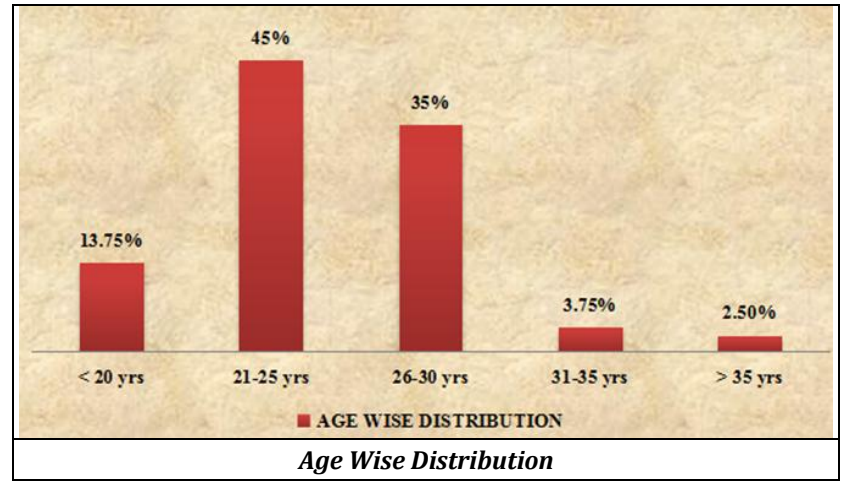

\begin{tabular}{|c|c|c|}
\hline Gestational Age (in Weeks) & No. of Patients & Percentage \\
\hline$<28$ weeks & 3 & $3.75 \%$ \\
\hline $28-32 \mathrm{wks}$ & 13 & $16.25 \%$ \\
\hline $32-36 \mathrm{wks}$ & 54 & $67.5 \%$ \\
\hline$>36 \mathrm{wks}$ & 10 & $12.5 \%$ \\
\hline Total & $\mathbf{8 0}$ & $\mathbf{1 0 0} \%$ \\
\hline \multicolumn{2}{|c|}{ Gestational Age at Diagnosis } \\
\hline
\end{tabular}
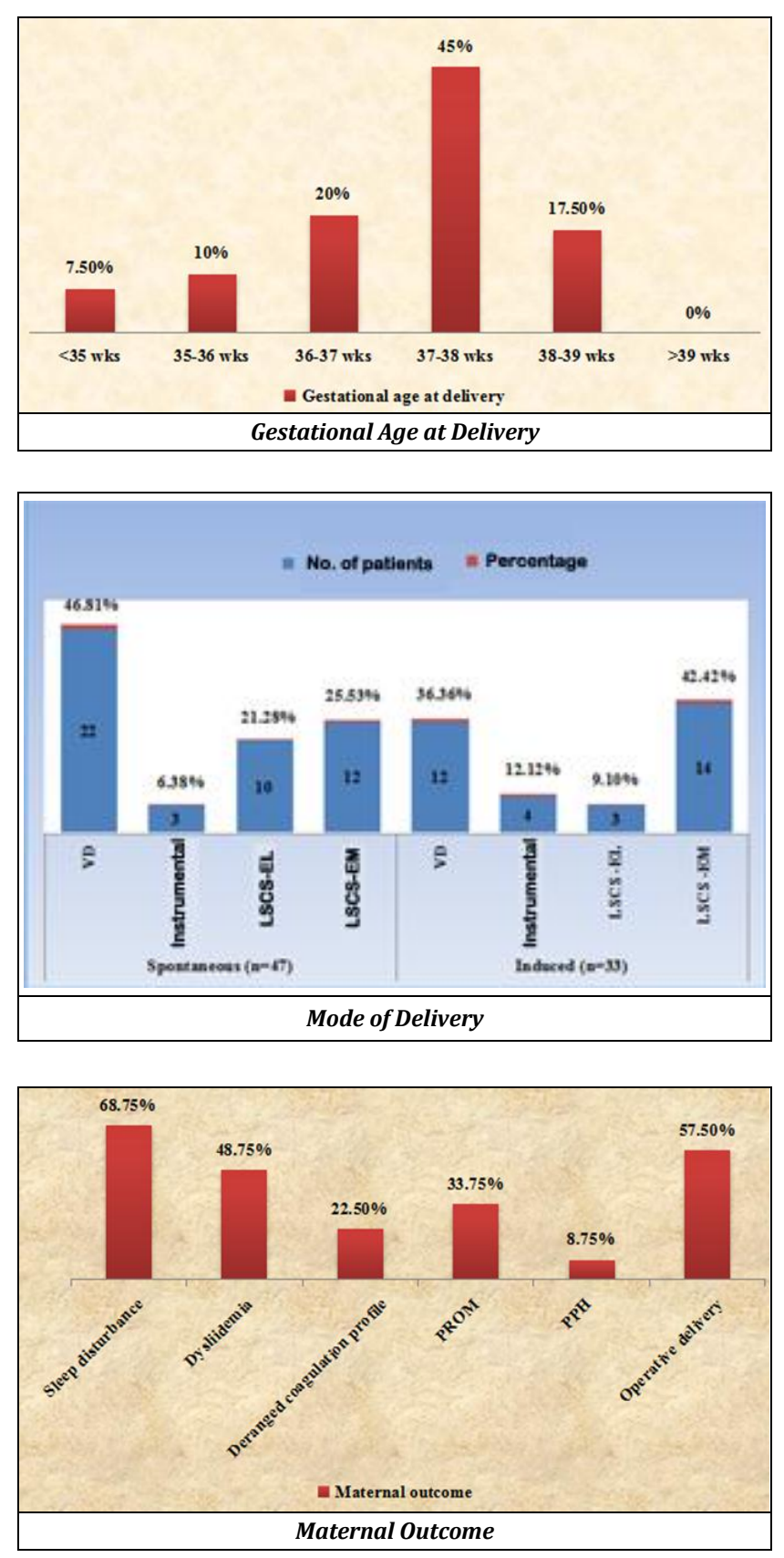

\begin{tabular}{|c|c|c|}
\hline Post-Partum Resolution & No. of Patients & Percentage \\
\hline YES & 78 & $97.5 \%$ \\
\hline NO & 2 & $2.5 \%$ \\
\hline Post-Partum Resolution of LFT (after 6 Wks. of Delivery) \\
\hline
\end{tabular}

\begin{tabular}{|c|c|c|}
\hline Foetal Outcome & Number & Percentage \\
\hline MSL & 19 & $23.75 \%$ \\
\hline Foetal distress & 16 & $20 \%$ \\
\hline Preterm birth & 20 & $25 \%$ \\
\hline LBW & 15 & $18.75 \%$ \\
\hline NICU admission & 15 & $18.75 \%$ \\
\hline IUFD/stillborn & 2 & $2.5 \%$ \\
\hline \multicolumn{2}{|c|}{ Distribution of Foetal Outcome } \\
\hline
\end{tabular}

The total number of deliveries during study period was 3609 , among them 183 patients had Obstetric Cholestasis according to inclusion and exclusion criteria. So, the incidence is $5 \%$.

The mean age (mean \pm S.D.) of the patients was $24.95 \pm$ 3.68 years with range $18-44$ years. Most of the patients (45\%) were at age between 21-25 years which was significantly higher than other age groups, like between 26 30 years and $<20$ years $(\mathrm{p}<0.0001)$. Only $3.75 \%$ of the patients were at age between 31-35 years and 2.5\% were at age $>35$ years.

In 80 patients, $48(60 \%)$ were primigravida and $32(40 \%)$ were multigravida. So the occurrence was seen more in primigravida than in multigravida $(\mathrm{p}<0.05)$. Most of the patients $(67.50 \%)$ diagnosed between $32-36$ weeks followed by $28-32$ weeks $(16.25 \%)$ which was significant $[\mathrm{p}$ - value $\{\mathrm{p}$ $<0.0001\}$ ]. $3.75 \%$ and $12.5 \%$ patients were diagnosed at $<28$ weeks and $>36$ weeks of gestation respectively.

$68.75 \%$ of the patients had sleep disturbance which was significant $(\mathrm{p}<0.0001) .22 .5 \%$ of the patients had deranged coagulation profile $(\mathrm{p}<0.0001)$. Mostly they had increased PT INR. PROM was found in $23.75 \%$ of cases in our study $(p<0.0001)$. The mean gestational age of delivery (mean \pm S.D.) of the patients was 37 weeks \pm 1 day with range $33-40$ weeks. Most of the patients (45\%) were delivered between 37-38 weeks $(\mathrm{p}<0.0001)$; followed by 36-37 weeks and 38-39 weeks, in which $20.0 \%$ and $17.50 \%$ patients delivered, respectively. Only $7.5 \%$ patients delivered before 35 weeks of gestation. In present study spontaneous onset of labour is seen in 47 (58.75\%) patients whereas induction is done in 33 $(41.25 \%)$ patients. In spontaneous group, most of the patients had VD (46.81\%) and LSCS was done in $46.81 \%$ of cases out of which $25.53 \%$ and $21.28 \%$ were elective and emergency respectively, and $6.38 \%$ of cases had instrumental delivery.

In the induced group most patient underwent LSCS (51.52\%) followed by vaginal delivery (36.36\%). Instrumental delivery was seen in $12.12 \%$ of cases. Although operative delivery in present study was more than vaginal delivery but the results were not significant $(p>0.05)$.

In our study among 80 cases of obstetric cholestasis, 7 $(8.75 \%)$ patients had postpartum haemorrhage during delivery and early puerperium while 73 patients did not experience postpartum haemorrhage $(91.25 \%)(\mathrm{p}<0.0001)$.

In most of the cases, postpartum resolution of LFT (97\%) was found $(\mathrm{p}<0.01)$ after 6 weeks of delivery and no symptoms found $(\mathrm{p}<0.0001)$.

In foetal outcome, Meconium Stained Liquor was found in $23.75 \%$ of the cases $(p<0.0001)$, foetal distress in $20 \%$ $(\mathrm{p}<0.0001)$. LBW in $18.75 \%(\mathrm{p}<0.0001)$. In $18.75 \%$ of the cases NICU admission were required due to low birth weight, 
foetal distress and meconium aspiration $(\mathrm{p}<0.0001)$. IUFD or Still Born was found only in $2 \%$ of the cases $(\mathrm{p}<0.0001)$.

\section{DISCUSSION}

Intrahepatic cholestasis of pregnancy (ICP) is the most common liver disease seen in pregnancy. ${ }^{10}$ It is typically a reversible cholestatic disease presenting in the second to third trimester of pregnancy and is characterized by pruritus predominantly of the palms and soles, elevated serum aminotransferases and/or elevated serum bile acid levels ( $>$ or $=10 \mathrm{micromol} / \mathrm{L}$ ) with spontaneous relief of laboratory abnormalities and symptoms promptly after delivery but no later than one month post-partum.

OC has been observed in almost all ethnic groups, but there is relevant geographical variation in its incidence. Incidence of $\mathrm{OC}$ in present study is $5 \%$. Padmaja et ${ }^{11} \mathrm{al}$ and $\mathrm{Ge}$ et al found highest incidence of $8.2 \%$ and $6.5 \%$ respectively whereas Hafeez et al,12 Sohail et al,13 Furrer et al ${ }^{14}$ and Rook et $\mathrm{al}^{15}$ found lower incidence of $\mathrm{OC}$ that is $3.1 \%, 2.8 \%, 2.3 \%$ and $1.9 \%$ respectively. The cause of so much variation from present study is may be due to geographical variation, ethnicity, environmental factor, different food habits or sample size calculation. More over our hospital is tertiary referral centre and hence incidence may be high due to referral of high risk pregnancies.

In OC, pruritus is intense and has specific predilection to palm and sole and mainly at night and hence pruritus leads to variable degree of sleep disturbances in patients with OC. The main presenting symptom in present study was generalised pruritus with varying degree of severity leading to sleep disturbance. Sleep disturbances in present study was $68.75 \%$ which is comparable to studies done by authors as Medda et $\mathrm{al}^{16}(60 \%)$ and Alakananda et $\mathrm{al}^{17}(65 \%)$. Skin rash and clinical jaundice was not noticed in any patients with OC. Serum bilirubin rarely exceeds more than $2 \mathrm{mg}$. Serum aminotransferases (ALT and AST) were raised from 2-10 fold but not above $1000 \mathrm{U} / \mathrm{L}$. Serum bile acid testing was not done in present study as it was not readily available to the patient and costly. UDCA was prescribed to patient along with topical emollients like calamine lotion to relief pruritus.

PROM was found in $23,75 \%$ of cases. The reason why PROM occurs in ICP is not known. The finding was consistent with Sinha et al ${ }^{18}(20 \%)$. But other studies like Ghimire et al ${ }^{19}$ $(10 \%)$ and Medda et al ${ }^{16}(10 \%)$ found lower incidences of PROM whereas Fawad et $\mathrm{al}^{20}(47 \%)$ found highest incidence of PROM.

Mean gestational age at delivery in present study was $37 \mathrm{~W} \pm 1 \mathrm{~d}$ which was comparable to studies by authors Rook et $\mathrm{al}^{15}(37 \mathrm{~W})$, Medda et $\mathrm{al}^{16}(37.28 \mathrm{~W})$, Alakananda et $\mathrm{al}^{17}$ $(37+1.7 \mathrm{~W})$. In our hospital, we followed the patient with weekly or fortnightly LFT on the basis of values of $\mathrm{Sr}$ Transaminases and if decreasing trend of LFTs noticed, then patient was followed till 38-39 weeks and then induction planned. If other associated factor like PIH, GDM or twin pregnancy or no improvement or increasing levels of LFTs was found, then induction was planned at 37-38 weeks after explaining the complication that might occur due to elective early intervention.

In present study spontaneous onset of labour was found in $58.75 \%$ of cases and induction was done in $41.25 \%$ of cases which was comparable to that observed by Mahajan et $\mathrm{al}^{21}(50.67 \%)$, Hak et $\mathrm{al}^{22}(50.67 \%)$ and Ghimire et $\mathrm{al}^{19}$ (51.25\%).

Vaginal delivery in both spontaneous and induced cases occurred in $51.25 \%$ of cases which comprises of $42.5 \%$ Normal delivery and $8.75 \%$ instrumental delivery. The rate of LSCS was $48.75 \%$ which comprises of $16.25 \%$ elective LSCS done for either previous LSCS, multiple gestation or Primi with breech pregnancy and $32.5 \%$ of emergency LSCS which was done either for failed induction, non-progress of labour, MSL and foetal distress. Although the rate of operative delivery was high but results are statistically not significant. The results of present study is comparable to the observations made by Sultana et $\mathrm{al}^{23}$ and Ghimire et al ${ }^{19}$ (VD $53.75 \%$, LSCS $46.25 \%$ ). Sultana et $\mathrm{al}^{23}$ found $46.37 \%$ had vaginal delivery and $13.33 \%$ and $40 \%$ cases underwent instrumental delivery and LSCS respectively.

In present study, alteration in coagulation profile was $22.5 \%$ which was comparable to that of Medda et al (19\%). Deranged coagulation was evaluated as raised PT INR in present study. All patients with deranged coagulation were given Vitamin $\mathrm{K}$ injection on three consecutive days and few patients in whom Sr. Bilirubin was raised, FFP was administered. The rate of PPH in present study was $8.75 \%$ which is comparable to those observed by Medda et al ${ }^{16}$ $(10 \%)$ and Ghimire et al ${ }^{19}(11.25 \%)$ whereas Dodampahala et $\mathrm{al}^{24}(29.6 \%)$ and Mahajan et al ${ }^{21}(16 \%)$ observed higher rates of PPH. PPH was less in present study due to active management of ICP cases with earlier UDCA prescription and administration of Vit K injection and FFP in some cases.

In present study, MSL was noticed in $23.75 \%$ of cases which is consistent with findings of Sultana et al (20\%) where as other authors i.e. Sinha et al (32.5\%), Ghimire et al $(32.5 \%)$ and Medda et al (42\%) found higher incidence of MSL and Sharma et al (8.33\%) found lower incidence of MSL. This variability may be due to study population taken from regular antenatal clinic and early intervention in form of UDCA. $20 \%$ had foetal distress in terms of foetal bradycardia or foetal heart rate decelerations which is consistent with findings of Medda et $\mathrm{al}^{16}$ (23\%), Ghimire et al ${ }^{19}(26.25 \%)$ and Sinha et $\mathrm{al}^{18}(17.5 \%)$. Sultana et $\mathrm{al}^{23}$ found higher incidence of MSL i.e. 33.3\% may be because they studied unbooked cases. In ICP, bile acid causes colonic motility stimulation leading to passage of meconium and foetal distress also leads to hypoxia and passage of meconium. Few researchers found correlation of Serum bile acids with foetal complication but that was not elucidated in present study because of non-availability of $\mathrm{Sr}$ bile acid testing. Percentage of preterm birth in present study was found to be $25 \%$ with 16 cases delivered at $36-37$ weeks, 8 cases at 35-36 weeks and 6 cases at $<35$ weeks. The findings correlates well with studies like Padmaja et al ${ }^{11}$ (24.4\%), Sohail et al (25.8\%), Alakananda et $\mathrm{al}^{17}$ (23\%) and others. Preterm birth was mostly due to PROM and few elective induction because of complain of less foetal movement with altered S/D ratio in colour doppler in USG. LBW seen in $18.75 \%$ of cases in present study which was comparable to other studies like Sohail et al (17.1\%), Singla et $\mathrm{a}^{25}(21.5 \%)$ and Alakananda et $\mathrm{al}^{17}(23 \%)$ but Medda et $\mathrm{al}^{16}(32 \%)$ found higher LBWs rate which may be due to elective early induction in study by Medda et al $^{16}$. LBW was mostly due to preterm delivery, and association with other maternal factor like PIH and twin pregnancy may be the 
reason. In present study, the rate of NICU admission was $18.75 \%$ of cases which was comparable with other studies like Fawad et $\mathrm{al}^{20}(22 \%)$, Alakananda et al ${ }^{17}$ (21\%), Jain et al (17.85). Geenes et $\mathrm{al}^{26}$ found less NICU admission which may be due to good antenatal surveillance and better environmental and socio-demographic factor. NICU admission was mostly due to preterm birth with low birth weight babies, respiratory distress and few with meconium aspiration syndrome. Percentage of IUFD in present study was $2.5 \%$ which was consistent with other studies like Fawad et $\mathrm{al}^{20}(2.8 \%)$, Alakananda et $\mathrm{al}^{17}(2 \%)$, Medda et $\mathrm{al}^{16}(2 \%)$. One case of IUFD occurred in booked case admitted for induction for obstetric cholestasis with PIH with hyperuricemia and on second day of induction, foetal bradycardia noticed for which emergency LSCS was done but baby could not be revived and in other case unbooked patient came with complain of less FM with IUD for which she was admitted and investigated and previous antenatal records checked which showed that it was a case of ICP. The cause of foetal death in ICP is thought to be acute anoxia. Autopsies of still borns show signs of acute anoxia with serosal and pulmonary petechial bleeding without intra uterine growth retardation.

\section{CONCLUSIONS}

It is found that maternal complications like pruritus, sleep disturbance and risk of operative delivery are benign and don't have any significant health impact in future on women. But Foetal complications are considerable in the form of prematurity and associated low birth weight, meconium aspiration syndrome and NICU admission. Hence pregnant women with OC should be followed very vigilantly till delivery and elective early induction should be weighed considering the risk of prematurity and its further health impact on growth and development of child.

\section{Limitations}

Since this study was taken in tertiary hospital which deals with high risk pregnancy cases bit more, and on small number of patients, results cannot be extrapolated to general population. Because of frequent antenatal visits, LFT and PT INR testing, OC patients faced significant cost burden. Only UDCA was used in present study and no other drugs were studied.

\section{ACKNOWLEDGEMENT}

I thank Dr Kumari Archana consultant OBG for being my coguide and helping me.

\section{REFERENCES}

[1] Gabzdyl EM, Schlaeger JM. Intrahepatic cholestasis of pregnancy: a critical clinical review. J Perinat Neonatal Nurs 2015;29(1):41-50.
[2] Lammert F, Marschall HU, Glantz A, et al. Intrahepatic cholestasis of pregnancy: molecular pathogenesis, diagnosis and management. J Hepatol 2000;33(6):101221.

[3] Hay JE. Liver disease in pregnancy. Hepatology 2008;47(3):1067-76

[4] Bacq Y, Sapey T, Brechot MC, et al. Intrahepatic cholestasis of pregnancy: a French prospective study. Hepatology 1997;26(2):358-64.

[5] Kenyon AP, Piercy CN, Girling J, et al. Obstetric cholestasis, outcome with active management: a series of 70 cases. BJOG 2002;109(3):282-8.

[6] Ray A, Tata RJ, Balsara R. Cholestasis of pregnancy. J Obstet Gynecol India 2005;55:247-50.

[7] Brites D, Rodrigues CM, Cardoso MC, et al. Unusual case of severe cholestasis of pregnancy with early onset, improved by ursodeoxycholic acid administration. Eur J Obstet Gynecol Reprod Biol 1998;76(2):165-8.

[8] Geenes V, Williamson C. Intrahepatic cholestasis of pregnancy. World Journal of Gastroenterology 2009;15(17):2049-66.

[9] Dann AT, Kenyon AP, Wierzbicki AS, et al. Plasma lipid profiles of women with intrahepatic cholestasis of pregnancy. Obstet Gynecol 2006;107(1):106-14.

[10] Royal College of Obstetricians and Gynaecologists Obstetric cholestasis. Green-Top Guideline No 43. 2006.

[11] Padmaja M, Pal B, Gupta JK, et al. A study of obstetric cholestasis. J Obstet Gynaecol India 2010;60(3):225-31.

[12] Hafeez M, Ansari A, Parveen S, et al. Frequency of intrahepatic cholestasis of pregnancy in Punjab Pakistan: a single centre study. J Pakistan Med Assoc 2016;66(2):203-6.

[13] Sohail S, Dar LR, Munawar S. Perinatal outcome in pregnancy complicated by obstetric cholestasis. PJMHS 2016;10(1):262-4.

[14] Furrer R, Winter K, Schäffer L, et al. Postpartum blood loss in women treated for intrahepatic cholestasis of pregnancy. Obstet Gynecol 2016;128(5):1048-52.

[15] Rook M, Vargas J, Caughey A, et al. Foetal outcomes in pregnancies complicated by intrahepatic cholestasis of pregnancy in a Northern California cohort. PLoS One 2012;7(3):e28343.

[16] Medda S, Sengupta S, Palo U. A study of the outcome of pregnancy complicated by obstetric cholestasis. Int J Reprod Contracept Obstet \& Gynecol 2018;7(3):9961001.

[17] Alakananda, Bhattacharrya A, Kavita. Foeto-maternal outcome in intrahepatic cholestasis of pregnancy. Sch J App Med Sci 2016;4(10D):3837-41.

[18] Sinha K, Pandey S, Das CR. Maternal and foetal outcome in cholestasis of pregnancy. J Nepalgung Medical College 2016;14(1):32-5.

[19] Ghimire SP, Ghimire A, Jha GS, et al. Foeto-Maternal outcomes in intrahepatic cholestasis in pregnancy in a tertiary care centre in Eastern Nepal. Journal of Nobel Medical College 2016;5(1):20-5.

[20] Fawad S. Diagnosis and neonatal outcome in obstetric cholestasis. Pak J Physiol 2016;12(1):15-7.

[21] Mahajan N, Afzal A, Lone MI. Outcome of pregnancy complicated by obstetric cholestasis: a prospective study. Int J Sci Stud 2017;5(3):271-4. 
[22] Hak J, Sharma N. Maternal and perinatal outcome in patients with cholestasis of pregnancy. J K Science 2015;17(4):168-71.

[23] Sultana R, Sarwar I, Fawad A, et al. Neonatal outcome in obstetric cholestasis patients at Ayub Teaching Hospital Abbottabad. J Ayub Med Coll Abbottabad 2009;21(4):768.

[24] Dodampahala SH, Pieris H, Chandrasena LG, et al. Presence of obstetric cholestasis in mothers presenting with pruritus in pregnancy: in a low resource South Asian setting. Adv Reprod Sci 2016;4(2):37-45.
[25] Singla S, Aggarwal P, Mittal A. Study to show the effect of intrahepatic cholestasis of pregnancy on perinatal outcome in uncomplicated pregnancy. J Evolution Med Dent Sci 2016;5(69):4964-6.

[26] Geenes V, Chappell LC, Seed PT, et al. Association of severe intrahepatic cholestasis of pregnancy with adverse pregnancy outcomes: a prospective populationbased case-control study. Hepatology 2014;59(4):148291. 